Original Paper http://ajol.info/index.php/ijbcs http://indexmedicus.afro.who.int

\title{
Evaluation des conditions de production, de la qualité physico-chimique et microbiologique des cossettes de manioc (Manihot esculanta Crantz) dans la commune de Bassila (Nord-Bénin)
}

\author{
G. René DEGNON*, T. R. Christian KONFO, S. Euloge ADJOU et \\ Edwige Dahouenon-AHOUSSI
}

\begin{abstract}
Université d'Abomey-Calavi, Ecole Polytechnique d'Abomey-Calavi, Laboratoire d'Etude et de Recherche en Chimie Appliquée, 01 BP 2009 Cotonou, Bénin.

*Auteur correspondant,E-mail: gnimabou2002@yahoo.fr
\end{abstract}

\section{REMERCIEMENTS}

Les auteurs remercient toutes les femmes productrices de cossettes de manioc ainsi que les agents des Secteur Communal du Développement Agricole (SCDA) de leurs contributions inestimables à la réalisation de cette recherche.

\section{RESUME}

La haute périssabilité des tubercules de manioc rend obligatoire leur transformation en différents produit dont les cossettes. L'objectif de cette étude est d'évaluer la qualité physico-chimique et microbiologique ainsi que les conditions de production des cossettes de manioc dans la commune de Bassila au Nord du Bénin. Ces conditions de production ainsi que les différentes variantes technologiques ont été évaluées à l'aide d'une enquête semi-structurée. Un échantillonnage aléatoire simple de 27 prélèvements regroupés en 09 échantillons a été ensuite fait. Les cossettes collectées ont été caractérisées aux plans physico-chimique et microbiologique. Les résultats ont révélé que les femmes productrices sont en majorité des groupes socioculturels et sociolinguistiques Koula et Kotokoli. Deux variantes technologiques de production ont été répertoriées. Ces procédés permettaient d'obtenir des cossettes de granulométries différentes. L'analyse des conditions de production a révélé des manquements graves aux règles élémentaires d'hygiène. Les échantillons collectés présentaient des teneurs en eau comprises entre $11,15 \pm 1,13 \%$ et $12,89 \pm 1,08 \%$ et trois échantillons sur les 09 ont eu une teneur en eau supérieure au critère de la Norme Béninoise (12\%). Tous les échantillons analysés sont de qualité microbiologique non satisfaisante. Il devient alors urgent de penser à l'amélioration des procédés de production des cossettes en vue de l'amélioration de la qualité des produits finis.

(c) 2018 International Formulae Group. All rights reserved.

Mots clés: Tubercules, manioc, transformation, cossettes, Bénin.

\section{Evaluation of the production conditions, the physico-chemical and microbiological quality of cassava (Manihot esculanta Crantz) chips in the commune of Bassila (Northern Benin)}




\begin{abstract}
High perishability of cassava roots impose their transformation into different products, including chips. This study aimed at evaluating the physico-chemical and microbiological quality and the conditions of production of cassava chips in Bassila (in Northern Benin). The conditions of production and different technological variants were assessed using a semi-structured survey. A simple random sample of 27 specimens grouped in to 09 samples was then made. The chips collected were characterized on physico-chemical and microbiological plans by standard methods. The results reveal that producers were women, from Koula and Kotokoli socio-cultural and sociolinguistic groups. Two technological variants of chips production were recorded and those just differed in cutting operations for one and crumbling to the other; which allowed obtaining different size chips. The analysis of production conditions has revealed serious breaches of basic hygiene. Evaluation of storability showed that the collected samples water contents were between $11.15 \pm 1.13$ and $12.89 \pm 1.08$ and that three samples in the 09 had exceeds Benin Standard. The evaluation of the microbiological quality of the samples showed that no samples satisfy the normative criteria. It becomes urgent to think about improving chips production processes to improve the finished products quality.
\end{abstract}

(C) 2018 International Formulae Group. All rights reserved.

Keywords: Tubers, cassava processing, chips, Benin.

\section{INTRODUCTION}

L'agriculture constitue une ressource territoriale historique majeure qui contribue au bien-être humain en produisant, entre autres, de la nourriture, des matières premières et de l'emploi (Abou et al., 2018 ; Millogo et al., 2018). Les plantes à racines, notamment le manioc, tiennent une place importante dans l'alimentation des béninois (Biaou et al., 2006). Le manioc s'impose désormais comme culture de première importance dans les nouvelles stratégies de développement agricole au Bénin. Grâce au Programme de Développement de la Filière Manioc (PDFM), la filière manioc se retrouve parmi les 13 filières identifiés (du point de vue de ses opportunités : les dérivés) à travers le plan stratégique de développement du secteur agricole. Ainsi, un important investissement est fait dans la filière manioc depuis quelques années. En conséquence, il a été enregistré une augmentation considérable de la production et de la transformation du manioc au Bénin. Ainsi, 3.603.019 tonnes de manioc ont été produites en 2015 contre 3.880.489 tonnes en 2014. La production des racines et tubercules à dominance manioc et igname a enregistré un accroissement de $14,14 \%$ et est passée de 6.128.288 tonnes en 2015 à 6.994.622 tonnes en 2016 (MAEP, 2017). De par l'accroissement de sa production, la diversité de ses dérivés et l'importance de sa demande sur les marchés nationaux et internationaux, le manioc constitue une culture socialement et économiquement porteuse.

A cause de l'importance des pertes poste-récoltes du manioc, sa transformation s'impose comme la meilleure stratégie de sa valorisation. Le manioc est généralement transformé en divers produits (gari, tapioca, farine, alcool, cossette etc.) dont la conservation est relativement facile. Cela se justifie par le fait qu'il contient près de $70 \%$ d'eau (Soulé et al., 2008 ; Diallo et al., 2013). Cette teneur élevée ne permet pas une longue conservation dans les conditions climatiques tropicales du Bénin.

Une enquête effectuée en 2013 auprès des 51 des 64 unités de transformations artisanales et semi artisanales montrent que ces dernières ont traité environ 4700 tonnes de manioc frais. Les $43 \%$ de ce volume de manioc frais ont été transformés par ces 51 unités, soit 43\% (2021 tonne) sont utilisés pour le gari, 7\% (329 tonnes) sont affectés au tapioca, 24\% (1128 tonnes) ont servi à la fabrication des cossettes et $2 \%$ (94 tonnes) sont destinés à la fabrication du lafun (Soulé et al., 2013). Les cossettes sont obtenues par épluchage, rouissage, fermentation et séchage des tubercules de manioc. Il a été rapporté qu'au Bénin, plusieurs technologies traditionnelles présentent des insuffisances 
pouvant influencer la qualité des produits finis (Konfo et al., 2014). C'est le cas de la transformation des tubercules de manioc en cossettes. Au fil du temps, les conditions de production et de stockage des cosettes n'auraient pas évolué. Le séchage des tubercules se ferait toujours en plein air, en bordure des voies. De plus, le processus de production souffre d'un manque de bonnes pratiques d'hygiène. Ainsi, les produits issus de cette transformation seraient très hétérogènes et répondraient difficilement aux critères fixés par la Norme Béninoise.

Cette étude avait pour objectif d'évaluer la qualité physico-chimique et microbiologique ainsi que les conditions de production des cossettes de manioc dans la commune de Bassila au Nord du Bénin.

\section{MATERIEL ET METHODES}

\section{Cadre d'étude}

L'enquête semi-structurée ayant permis de répertorier les variantes technologiques de production de cossettes a été réalisée dans neuf (09) villages de l'arrondissement d'Allédjo dans la commune de Bassila (Latitude : 9 $9^{\circ} 00^{\prime} 29^{\prime \prime}$ Nord/Longitude : 1 ${ }^{\circ} 39^{\prime} 55^{\prime \prime}$ Est). Le choix de ces villages se justifie par le fait qu'ils sont des zones de forte production de cossettes dans la commune. Des interviews semistructurées et structurées, individuelles et de groupe avec les transformatrices ont été effectuées. Les informations collectées étaient relatives aux données socioculturelles et sociolinguistiques, le niveau d'instruction, la technologie de production... Des fiches d'enquête ont été également adressées aux agents en service au Secteur Communal du Développement Agricole (SCDA) de Bassila. A cet effet nous avons réalisé 50 fiches d'enquêtes qui ont été adressées aux structures et aux personnes ciblées.

\section{Evaluation de la qualité physicochimique et microbiologique des cossettes de manioc collectées \\ Echantillonnage}

Afin d'évaluer les qualités physicochimique et microbiologique des cossettes de manioc produites dans les localités enquêtées, un répertoire de productrices connus dans chaque village a été constitué. Ensuite, un échantillonnage aléatoire simple de trois productrices a été effectué par localité à l'aide du logiciel Microsoft Excel. Au total, 27 prélèvements ont été effectués dans les neuf (09) villages (Alédjo, Alédjo, Akaradè, Partogo, Tchémbéri, Kadagué, Boutou, Kaouté, Odola, et Nibadara). Les prélèvements issus de chaque localité ont été ensuite soigneusement mélangés pour constituer un total de neuf (09) échantillons qui ont fait l'objet des analyses physicochimiques et microbiologiques.

\section{Caractérisation physico-chimique des échantillons}

La matière sèche et le $\mathrm{pH}$ ont été déterminés par la méthode AOAC (1984). L'acidité exprimée en gramme par litre $(\mathrm{g} / \mathrm{L})$ a été déterminée par dosage avec de l'hydroxyde de sodium $0,1 \mathrm{~mol} / \mathrm{L}$ en présence de la phénolphtaléine comme indicateur coloré (AOAC, 1990).

\section{Caractérisation microbiologique des échantillons}

Les échantillons prélevés ont été évalués en recherchant par des méthodes standards, les paramètres microbiologiques de qualité. Ainsi, la flore aérobie mésophile totale a été dénombrée par ensemencement sur le milieu Plate Count Agar (PCA) et incubation à $30^{\circ} \mathrm{C}$ pendant 24-48 h (NF V08051), alors que les coliformes totaux, thermotolérants ont été recherché sur le milieu Violet Red Bile Lactose (VRBL) avec incubation à 30 et $44{ }^{\circ} \mathrm{C}$ respectivement pendant $24 \mathrm{~h}$ (NF V08-050). Quant à E. coli une à deux öses du milieu VRBA sont inoculées sur un milieu EMB et l'incubation a été effectuée pendant $24 \mathrm{~h}$ à $37^{\circ} \mathrm{C}$ (NF ISO 7251). Staphylococcus aureus a été recherché sur le milieu Baird Parker avec incubation à $37{ }^{\circ} \mathrm{C}$ pendant $24-48$ (NF EN ISO 68881/A1), tandis que les levures et moisissures l'ont été sur le milieu Sabouraud au chloramphénicol, puis incubées à $25{ }^{\circ} \mathrm{C}$ pendant 3 à 5 jours (NF V08-059). Les Bactérie Anaérobies Sulfito-Réducteurs ont été recherché sur gélose Tryptone Sulfite Néomycine (TSN) avec incubation à $46{ }^{\circ} \mathrm{C}$ pendant 20H (NF ISO 15213). Enfin la 
recherche des Salmonelles a été effectuée par un pré-enrichissement de la solution mère à 37 ${ }^{\circ} \mathrm{C}$ pendant $19 \mathrm{~h}$. L'enrichissement a été fait sur les milieux Rappaport Vassiliadis (RV), Müller Kaüffmann (KM) et l'isolement sur les milieux Xylose-Lysine-Décarboxylate (XLD) et Hektoen (ISO 6579).

\section{Analyses statistiques}

Les données issues de cette recherche ont été soumises à une statistique descriptive. Ainsi, les valeurs moyennes ainsi que les écart-types ont été calculés. Ces données ont été organisées sous forme de tableaux et de graphiques à l'aide du tableur Microsoft Excel 2010.

\section{RESULTATS}

\section{Conditions de production des cossettes de manioc}

Profils socio-culturel et socio-linguistique des productrices

Dans cette partie des résultats, nous présentons les données sociales relatives aux productrices de cossettes, les variantes technologiques de production des cossettes et les conditions de production des cossettes.

Les résultats issus de la classification des productrices en tenant compte de leur répartition selon les groupes socio-culturels et socio-lingustiques, l'âge et le niveau d'instruction ont été présentés dans les figures 1,2 et 3. L'analyse de ces résultats a montré que la production et la commercialisation des cossettes étaient plus pratiquées (Figure 3) par les groupes socio-culturels et sociolingustiques Koura $(75,25 \%)$ et Kotokoli $(23,73 \%)$. Les productrices sont de la trentaine à la quarantaine d'âge en majorité $(38,4 \%)$ en moyenne (Figures 1) avec un niveau d'instruction (Figure 2) primaire $(63,83 \%)$ ou non scolarisées $(36,17 \%)$.

Variantes technologiques de production de cossettes de manioc dans les localités ciblées

$\mathrm{Au}$ terme de l'enquête, deux technologies de productions ont été répertoriées (Figures 7 et 8). Ces deux technologies présentent beaucoup de similitudes. Après réception, les tubercules débarrassés des épluchures (parties contenant l'essentiel des cyanures et des tannins), sont trempés dans de l'eau additionnée d'inoculum dans des fûts hermétiquement fermés. L'inoculum utilisé pour cette opération est un ferment naturel obtenu à partir du jus de manioc trempé dans de l'eau durant $28 \mathrm{~h}$ environ.

La température ambiante est favorable pour la multiplication des micro-organismes responsables $\mathrm{du}$ ramollissement des tubercules. Ainsi, après 36 heures de trempage, on obtient des tubercules bien ramollis (au touché) qu'on doit sortir de l'eau pour leur faire subir la fermentation. Elle se fait par entassement des pâtes de tubercules ramollis dans des sacs surmontés de poids afin d'égoutter partiellement l'excès d'eau lors du rouissage. Selon le type de produit souhaité, on effectue ensuite un découpage (variante1) ou un émiettage (variante 2) par pilage (Figure 4-Photos A et B respectivement). Le pilage est effectué lorsqu'on veut obtenir un produit consommable après seulement deux jours de séchage.

\section{Conditions de production des cossettes de manioc dans les localités ciblées}

Toutes les transformatrices opèrent en plein air, parfois non loin des routes en latérite où d'énormes quantités de poussières se déposent chaque jour (Photos F). La qualité de l'eau (provenant des citernes ou des puits à cause des coupures régulières de l'eau de robinet) utilisée au cours de la transformation est parfois douteuse (Photo $\mathrm{C}$ ) et les produits (intermédiaires ou finis) sont entreposés par terre, dans un environnement où les animaux pullulent et les enfants s'amusent à longueur de journée (Photos D, E et F). En outre, d'autres productrices sèchent les cossettes sur des tôles de cuisines malpropres et à la portée des oiseaux, de la volaille et de la flore aéroportée (Photo G).

Caractéristiques physico-chimiques et microbiologique des cossettes produites dans les localités ciblées

Caractéristiques physico-chimiques des cossettes produites dans les localités ciblées

Les échantillons collectés présentent des teneurs en eau comprises entre 
$11,15 \pm 1,13 \%$ (Patargo) et $12,89 \pm 1,08 \%$ (Kaouté). Cette figure révèle également que les échantillons collectés à Nibadowa, à Odola et à Kaouté ont une teneur en eau supérieure à $12 \%$ (Figure 7).

Les teneurs en matière sèche calculées au cours de cette étude ont varié entre 88,83 $\pm 1,0 \%$ et $87,10 \pm 1,08 \%$. Les échantillons collectés à Alédjo, Tchémbéri, Boutou et Kadégué ont présenté les plus fortes teneurs en matière sèche (tableau1). Aussi, tous les échantillons ont un $\mathrm{pH}$ acide $(5,3 \pm 1,05$ et $6,51 \pm 03)$ et une acidité titrable plus ou moins

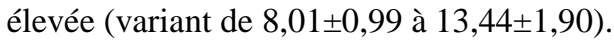

\section{Caractéristiques microbiologiques des} cossettes produites dans les localités ciblées

L'analyse du Tableau 2 révèle que les échantillons sont contaminés par les germes aérobies mésophiles, les coliformes totaux, les coliformes thermo-tolérants, staphylocoques coagulase positive, les levures et les moisissures. Par contre, les entérobactéries présumées, les bactéries Anaérobies Sulfito-
Réductrices (ASR) et les salmonelles sont absents dans tous les échantillons prélevés. $E$. coli glucuro-nidase+ est fortement présent dans les échantillons collectés à Nibadarou, à Kaouté, à Kadegue, à Partago à Tchemberi, à Aledjo et à Odola tandis que sa présence dans les autres échantillons est à des proportions indétectables.

Les proportions des échantillons répondant aux critères microbiologiques ont été présentées sur la figure 8 . Son analyse révèle qu'aucun des échantillons prélevés ne satisfait les critères normatifs relatifs à la flore aérobie mésophile totale, aux levures et moisissures, aux coliformes présumés, aux coliformes thermotolérants ainsi que pour Staphylococcus aureus. Néanmoins, tous les échantillons satisfont les critères relatifs aux Bactéries Sulfito-Réductrices, aux entérocbactéries présumés et aux Salmonelles. Enfin, seulement $33,33 \%$ des échantillons sont satisfaisants pour le dénombrement de Escherichia coli.

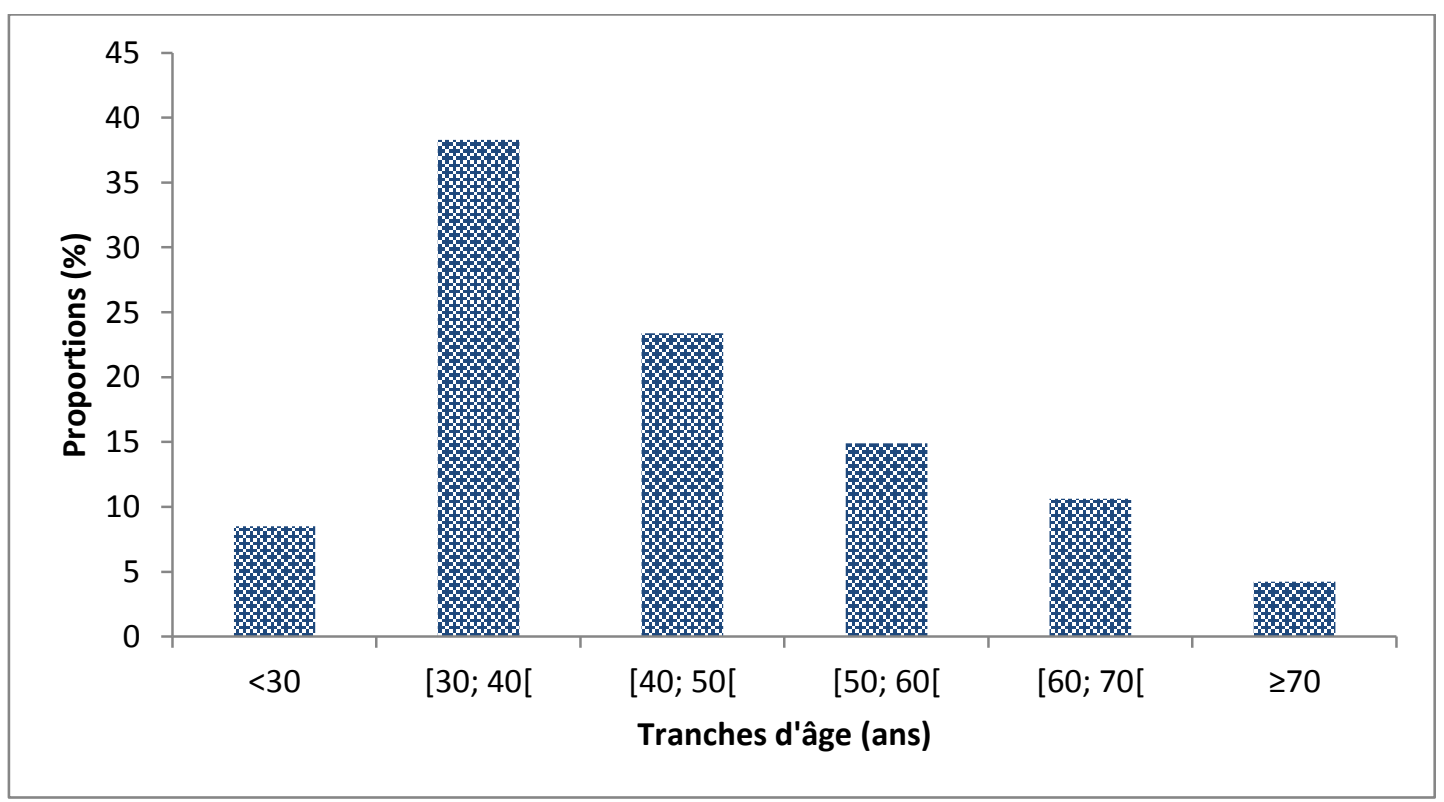

Figure 1 : Répartition des productrices en fonction de leurs âges. 


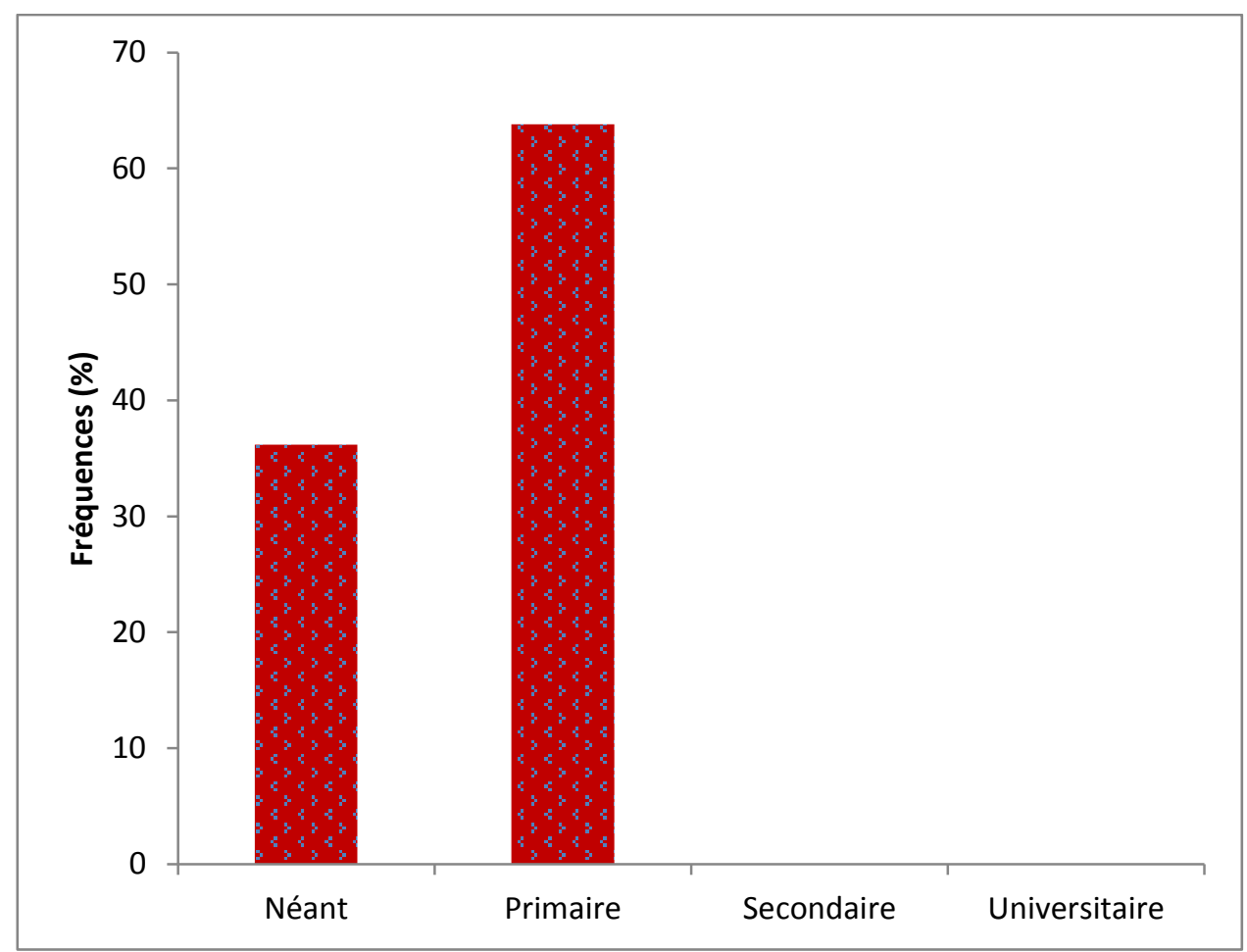

Figure 2 : Répartition des productrices en fonction de leur niveau instruction.

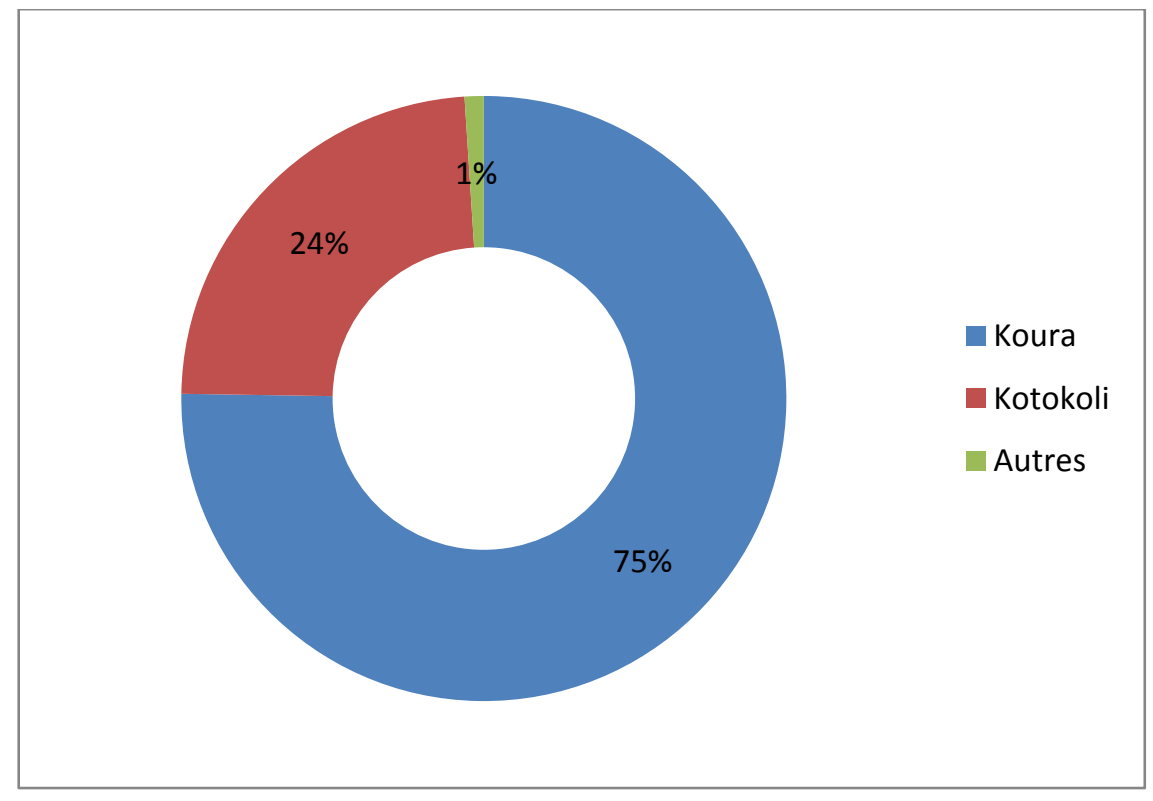

Figure 3 : Répartition des productrices en fonction de leurs groupes socioculturels et sociolinguistiques. 

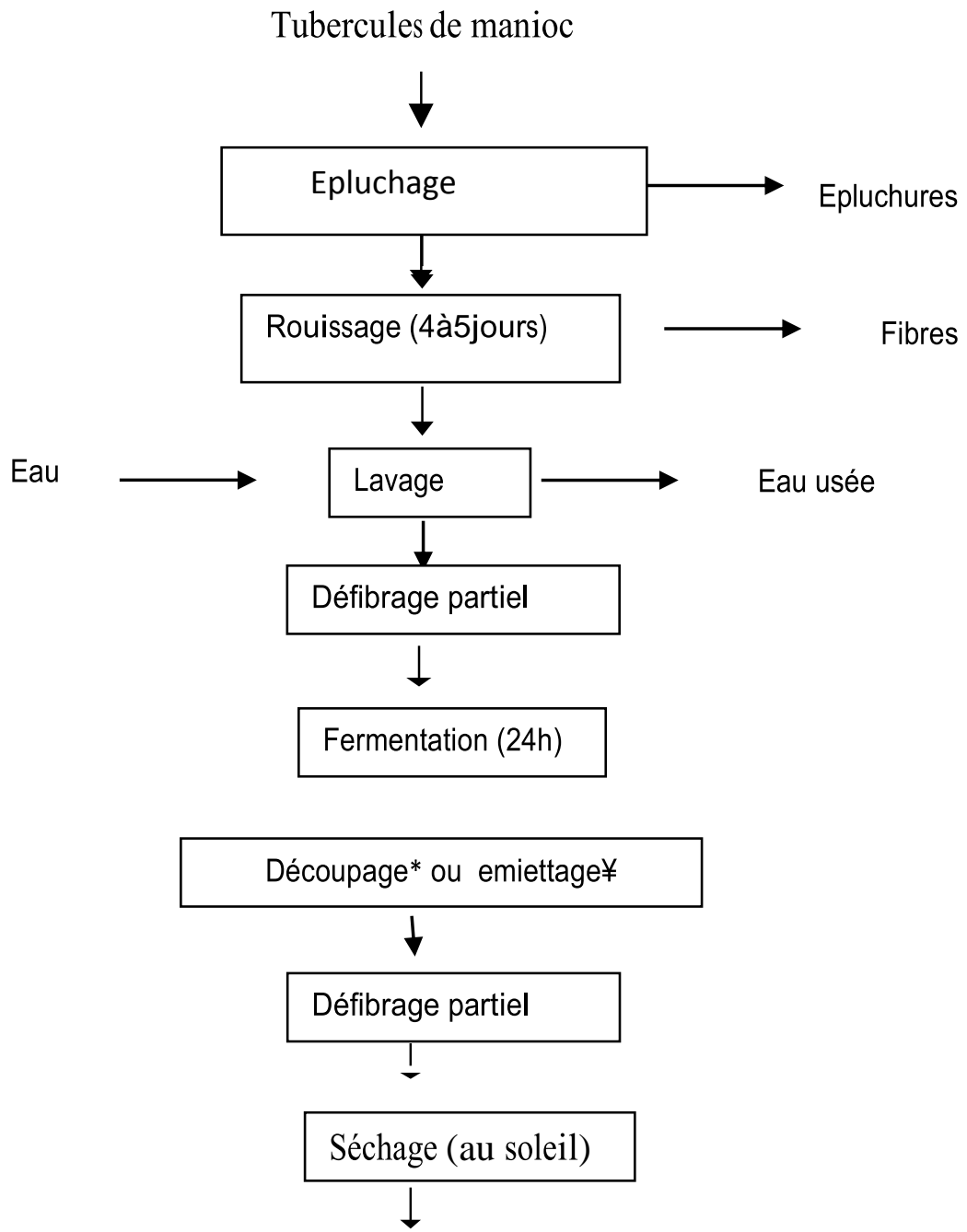

Cossettes de manioc

Figure 4: Transformation traditionnelle de tubercules de manioc en cossettes (variante 1).

* Variante 1 ; $¥$ Variante 2

Tableau 1: Teneurs en matière sèche, $\mathrm{pH}$ et acidité titrable des échantillons

\begin{tabular}{llll}
\hline $\begin{array}{l}\text { Paramètres } \\
\text { Localités }\end{array}$ & $\begin{array}{l}\text { Matière } \\
\text { Sèche }(\%)\end{array}$ & \multicolumn{1}{c}{$\mathbf{p H}$} & Acidité titrable $\mathbf{( g / L )}$ \\
\hline Boutou & $88,68 \pm 1,04$ & $5,3 \pm 1,05$ & $8,05 \pm 1,10$ \\
Nibadowa & $87,71 \pm 1$ & $5,40 \pm 0,99$ & $9,12 \pm 2,10$ \\
Patargo & $88,84 \pm 1,13$ & $5,50 \pm 0,88$ & $13,44 \pm 1,90$ \\
Odola & $87,99 \pm 0,95$ & $5,10 \pm 0,89$ & $12,40 \pm 1,00$ \\
Kaouté & $87,10 \pm 1,08$ & $5,90 \pm 0,55$ & $9,40 \pm 2,4$ \\
Kadégué & $88,49 \pm 0,77$ & $6,50 \pm 1,03$ & $8,01 \pm 0,99$ \\
Akaradè & $88,46 \pm 0,86$ & $5,90 \pm 1,01$ & $12,4 \pm 0,84$ \\
Aledjo & $88,83 \pm 1,03$ & $5,50 \pm 0,25$ & $13,1 \pm 1,41$ \\
Tchémberi & $88,80 \pm 0,99$ & $5,10 \pm 0,77$ & $11,4 \pm 0,85$ \\
\hline
\end{tabular}


Tableau 2 : Qualité microbiologique des échantillons collectés.

\begin{tabular}{|c|c|c|c|c|c|c|c|c|c|}
\hline \multirow[b]{2}{*}{$\begin{array}{l}\text { RECHERCHE } \\
\text { EFFECTUEE }\end{array}$} & \multirow[b]{2}{*}{$\begin{array}{l}\text { Flore } \\
\text { totale } \\
(\text { UFC /g) }\end{array}$} & \multirow[b]{2}{*}{$\begin{array}{l}\text { Entérobactéries } \\
\text { présumés UFC/g }\end{array}$} & \multicolumn{3}{|c|}{ COLIMETRIE UFC/G } & \multirow[b]{2}{*}{$\begin{array}{l}\text { Staphylocoques } \\
\text { aureus } \\
\text { UFC/g }\end{array}$} & \multirow[b]{2}{*}{$\begin{array}{l}\text { Bactéries Sulfito- } \\
\text { Réductrices } \\
\text { UFC/g }\end{array}$} & \multirow[b]{2}{*}{$\begin{array}{l}\text { Levures et } \\
\text { Moisissures } \\
\text { UFC/g }\end{array}$} & \multirow[b]{2}{*}{$\begin{array}{l}\text { Salmonelles } \\
\text { dans 25g de } \\
\text { cossette }\end{array}$} \\
\hline & & & $\begin{array}{l}\text { Coliformes } \\
\text { présumés } \\
\text { UFC/g }\end{array}$ & $\begin{array}{l}\text { Coliformes thermo- } \\
\text { tolérants } \\
\text { UFC/g }\end{array}$ & $\begin{array}{l}\text { E. coli } \\
\text { UFC/g }\end{array}$ & & & & \\
\hline NIBADAROU & $2,1.10^{6}$ & - & $>3.10^{4}$ & $>3.10^{3}$ & $>3.10^{3}$ & $>1,5.10^{4}$ & - & $\begin{array}{l}\mathrm{L}: 1,3 \cdot 10^{5} \\
\mathrm{M}: 1,3.10^{4}\end{array}$ & - \\
\hline KAOUTE & $>3.10^{6}$ & - & $>3.10^{4}$ & $>3.10^{3}$ & $>3.10^{3}$ & $>1,5.10^{4}$ & - & $\begin{array}{l}L>3.10^{5} \\
M: 4,2.10^{4}\end{array}$ & - \\
\hline KADEGUE & $>3.10^{6}$ & - & $>3.10^{4}$ & $>3.10^{3}$ & $2,5.10^{2}$ & $>1,5.10^{4}$ & - & $\begin{array}{l}\mathrm{L}: 4,7.10^{4} \\
\mathrm{M}: 7.10^{3}\end{array}$ & - \\
\hline PATARGO & $>3.10^{6}$ & - & $>3.10^{4}$ & $>3.10^{3}$ & $>3.10^{3}$ & $>1,5.10^{4}$ & - & $\begin{array}{l}\mathrm{L}>3 \cdot 10^{5} \\
\mathrm{M}: 4,7.10^{4}\end{array}$ & - \\
\hline TCHEMBERI & $2,6.10^{6}$ & - & $>3.10^{4}$ & $>3.10^{3}$ & $<10$ & $>1,5.10^{4}$ & - & $\begin{array}{l}L>9,2.10^{4} \\
M: 2.10^{3}\end{array}$ & - \\
\hline BOUTOU & $>3.10^{6}$ & - & $>3.10^{4}$ & $>3.10^{3}$ & $<10$ & $>1,5.10^{4}$ & - & $\begin{array}{l}L>1,3.10^{5} \\
M>1,5.10^{5}\end{array}$ & - \\
\hline ALEDJO & $>3.10^{6}$ & - & $>3.10^{4}$ & $>3.10^{3}$ & $>3.10^{3}$ & $>1,5.10^{4}$ & - & $\begin{array}{l}\mathrm{L}>3.10^{5} \\
\mathrm{M}: 8.10^{3}\end{array}$ & - \\
\hline ODOLA & $>3.10^{6}$ & - & $>3.10^{4}$ & $>3.10^{3}$ & $7.10^{2}$ & $>1,5.10^{4}$ & - & $\begin{array}{l}\mathrm{L}: 3,6.10^{4} \\
\mathrm{M}: 1,3.10^{4}\end{array}$ & - \\
\hline AKAREDE & $>3.10^{6}$ & - & $>3.10^{4}$ & $>3.10^{3}$ & $<10$ & $>1,5.10^{4}$ & - & $\begin{array}{l}\mathrm{L}: 3,6.10^{4} \\
\mathrm{M}: 1,3.10^{4}\end{array}$ & - \\
\hline $\begin{array}{r}\text { Critères : } \\
\mathrm{m} \\
\mathrm{M}\end{array}$ & $\begin{array}{l}10^{5} \\
10^{6}\end{array}$ & - & $\begin{array}{l}10^{3} \\
10^{4}\end{array}$ & - & - & - & - & - & - \\
\hline
\end{tabular}




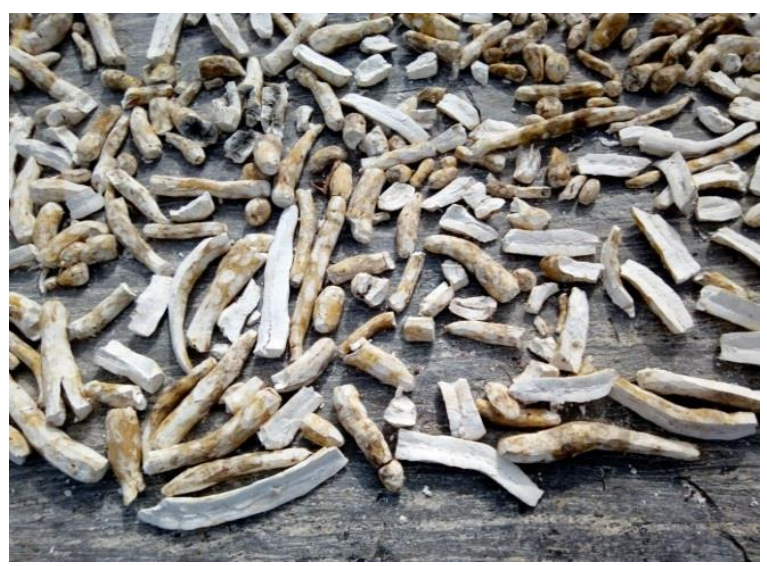

Photo A : Cossettes obtenues par découpage.

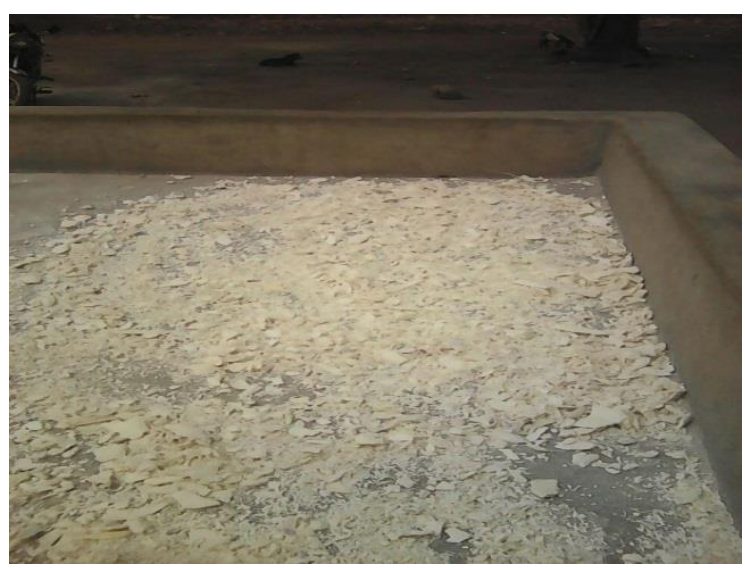

Photo B : Cossettes obtenues par émiettage.

Figure 5 : Photos de produits finis issus des différentes variantes de transformations.

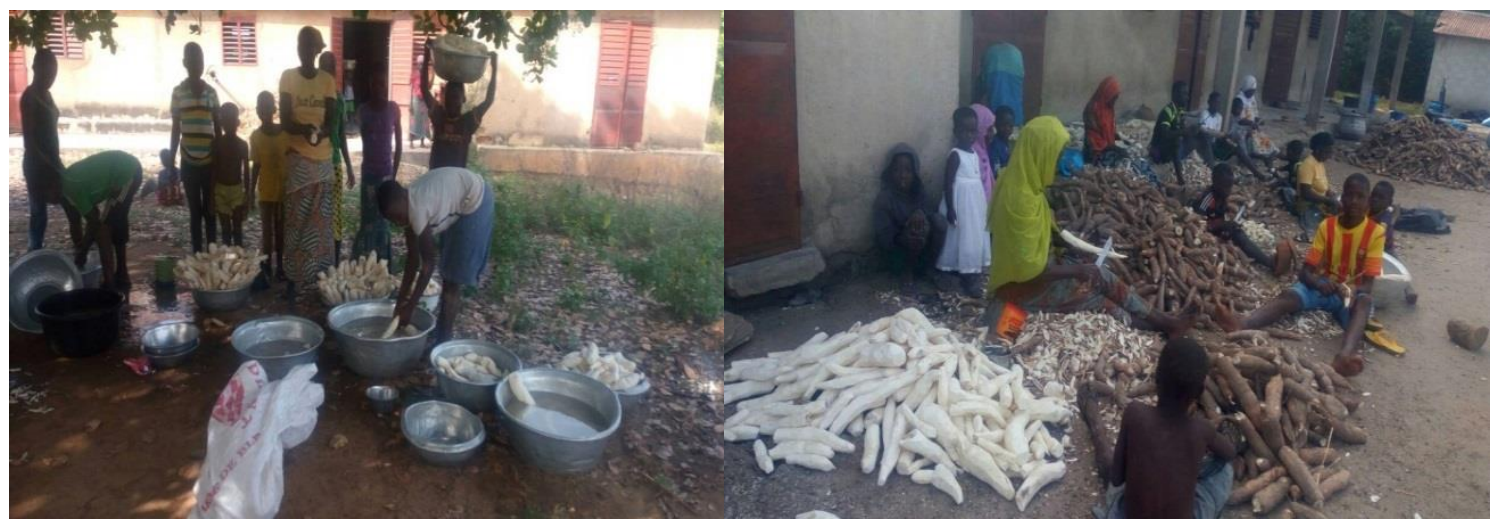

Photo $\mathbf{C}$ : Echantillon posés à même le sol dans un environnement malsain.

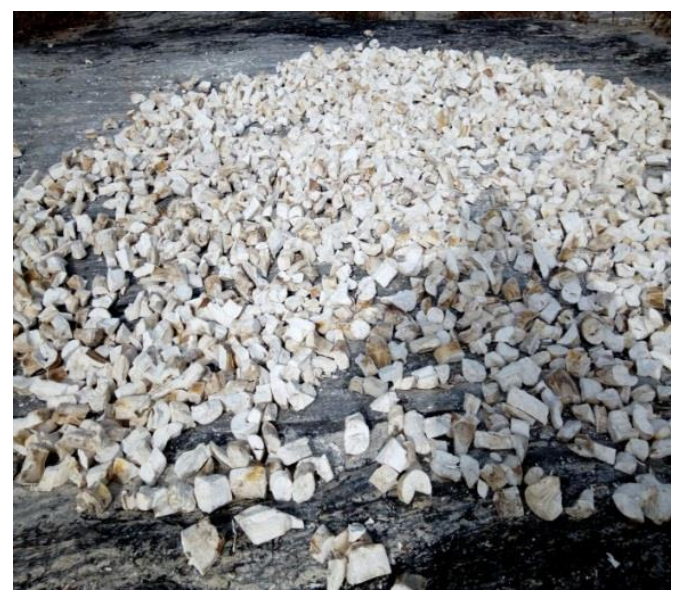

Photo E : Cossettes mises en séchage dans une aire naturelle (roche).
Photo D : Maniocs épluchés entreposés en vrac par terre.

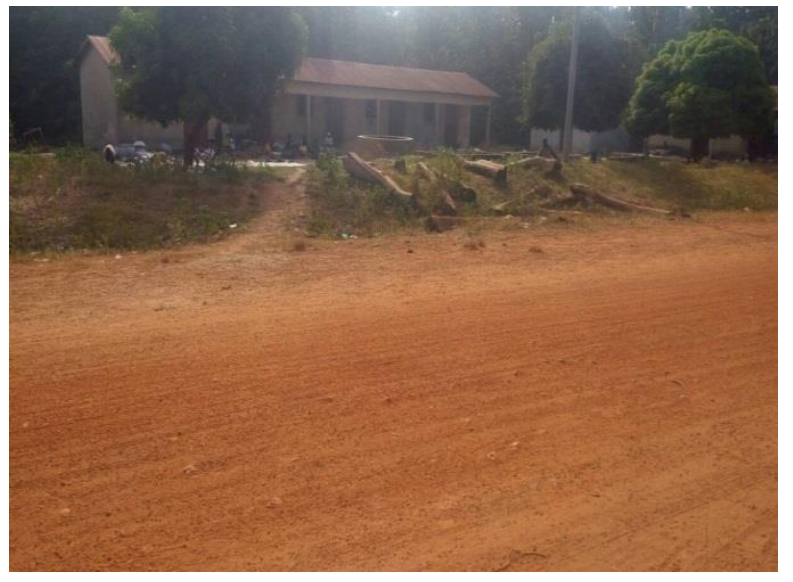

Photo F : Cossettes mises en séchage non loin d'une voie en latérite (poussière) et autour desquelles pullulent des animaux. 


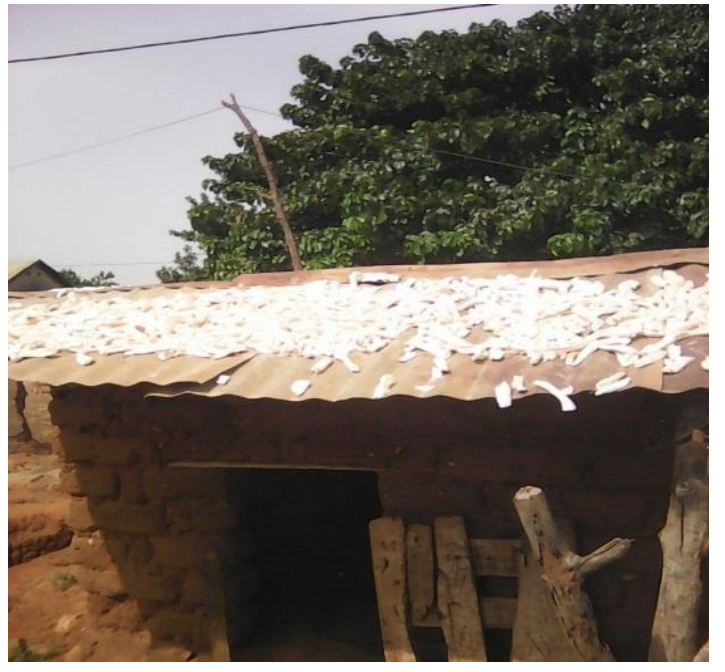

Photo G : Cossettes mises en séchage sur des tôles de cuisines.

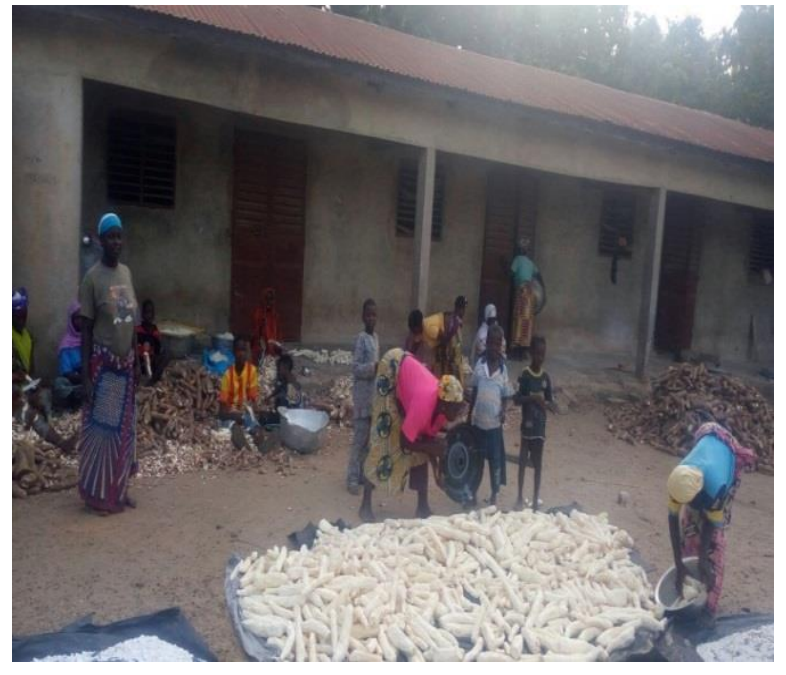

Photo G : Cossettes mises en séchage dans un environnement où les enfants s'amusent.

Figure 6 : Photos montrant les conditions de manque d'hygiène dans lesquelles les cossettes sont transformées.

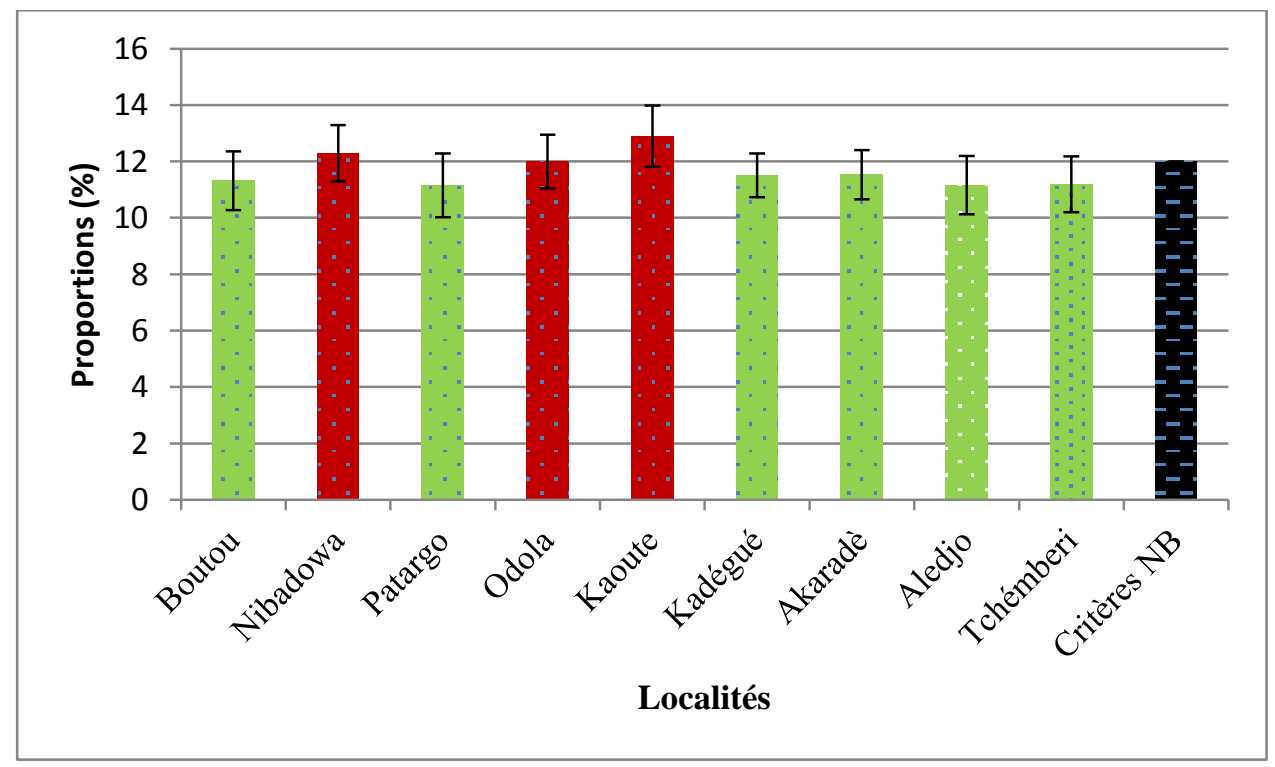

Figure 7 : Teneur en eau des échantillons de cossettes. 


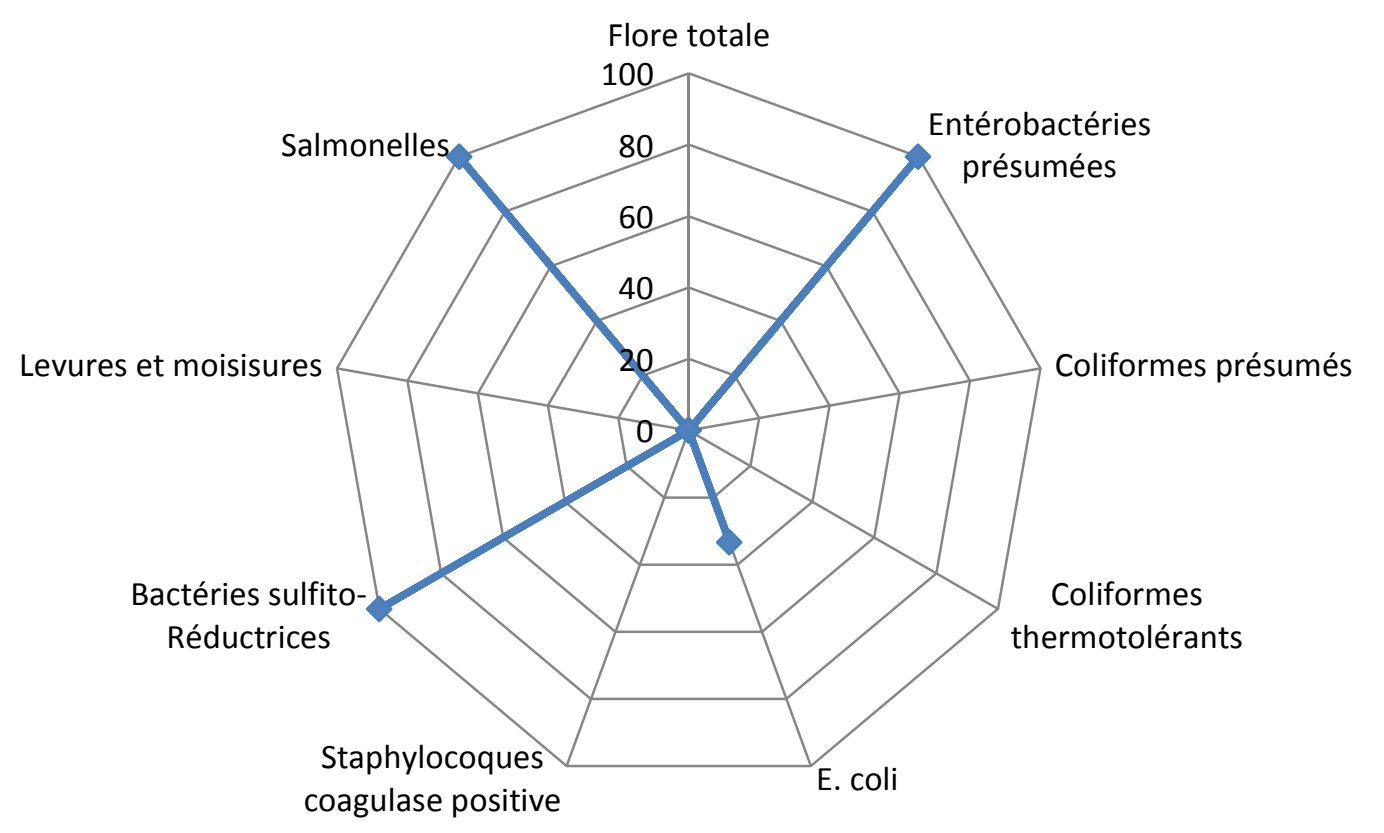

Figure 8 : Proportions des échantillons répondant aux critères microbiologiques.

\section{DISCUSSION}

Le manioc (Manihot esculenta Crantz) est une importante plante à tubercule cultivée dans de nombreux pays à travers le monde et plus particulièrement en Afrique de l'Ouest (Gmakouba et al., 2018). Deux variantes technologiques de production de cossettes de manioc ont été répertoriées dans cette étude. Ces procédés diffèrent seulement au niveau d'une opération unitaire à savoire le découpage (variante1) ou l'émiettage (variante 2). Ces procédés concordent avec ceux rapportés par Rasoanantoandro-Gothard (2003) en République Centre Africaine (RCA) qui a fait des investigations sur l'amélioration des techniques de transformation du manioc en cossettes. Ces travaux qui ont expérimenté le rouissage en vase clos et le séchage en cage thermique ont montré qu'en moins de 12 h, la température de $28{ }^{\circ} \mathrm{C}$ est atteinte. Celleci est favorable pour la multiplication des micro-organismes responsables du ramollissement des tubercules.
Les fortes teneurs en eau relevées dans les échantillons collectés à Nibadowa et Kaouté $(12,28 \%$ et $12,89 \%$ respectivement $)$ sont supérieurs au critère normatif $(<12 \%)$ rapportés par Soule et al., 2008). Ceci peut engendrer une faible aptitude à la conservation de ces cossettes. En effet, selon Faiveley, (2012), la teneur en eau des aliments est corrélée avec leur durée de conservation. Bien qu'elle n'apporte aucune valeur énergétique aux aliments, son existence joue un rôle très important. Elle influence la structure, l'apparence, le goût des aliments et leur susceptibilité à la dégradation. Elle impacte principalement sur l'activité enzymatique et la croissance microbienne dans l'aliment. La croissance des bactéries par exemple est généralement impossible lorsque l'activité de l'eau $\left(\mathrm{a}_{\mathrm{w}}\right.$ : paramètre fortement lié à la teneur en eau) est inférieur à 0,90 . Les moisissures et les levures sont inhibés respectivement vers une $a_{w}$ de 0,7 et 0,8 sauf certaines moisissures et levures osmophiles qui peuvent se développer jusqu'aux des $a_{w}$ de 0,6 . 
Tout ceci suggère qu'il faut optimiser l'opération de séchage comme l'ont déjà suggéré par Karidioula et al., (2018) pour les fèves de cacao. Les résultats de recherche sont similaires aux résultats $(84,47-88,63 \%)$ obtenus par Mukandila et al., (2010) sur la qualité des produits dérivés du manioc prélevés dans les marchés de Kinshasa (RD Congo). Cependant, la faible acidité observée au niveau des échantillons pourrait témoigner de l'activité des bactéries lactiques au cours de la fermentation.

D'autres part, la présence des moisissures sur les produits alimentaires dérivés du manioc, comme constaté par le présent travail, a été rapportée par d'autres études antérieures (Gnonlonfin et al., 2007). Amadi et Adebola (2008) ont constaté la présence de A. flavus et de A. niger dans du gari stocké au Nigeria. Essono et al. (2007) ont observé la présence de treize espèces de Aspergillus sur les cossettes de manioc au Cameroun. Au Sénégal, Diallo et al., (2013) ont remarqué la présence des Aspergillus spp. En ce qui est de la présence des germes aérobies mésophiles, le résultat obtenu par le présent travail confirme celui obtenu par Djoulde et al. (2007) au Bénin, GbaguidiDarboux (2001) a noté l'implication des bactéries dans le processus de fermentation du manioc. D'après Darman (2004), la flore microbienne sur les produits dérivés du manioc est constituée principalement par les bactéries lactiques et ont été identifiées lors de la fermentation du manioc pour la production du gari (Moorthy et Mathew, 1998). Ainsi, la flore aérobie mésophile constatée sur les produits dérivés du manioc dans ce travail serait principalement constituée par les bactéries lactiques.

Le niveau de contamination microbiologique élevé de certains échantillons par S. aureus, les champignons (levures et moisissures) ainsi que $E$. coli expose le consommateur à des affections d'origines alimentaires telles les infections, les toxiinfections et les intoxinations alimentaires (Boubendir, 2014). L'intoxination alimentaire staphylococcique est due à des entérotoxines produites par plusieurs espèces de Staphylococcus (principalement S. aureus).
Les toxines sont thermostables et ne sont pas inactivées par le chauffage ou la cuisson de l'aliment. Ces résultats microbiologiques non satisfaisants pourraient s'expliquer par les niveaux de scolarisation faible (primaire $(63,83 \%)$ ou non scolarisées $(36,17 \%))$ des productrices qui ont ainsi peu ou pas de notions des règles de Bonnes Pratiques d'Hygiène $(\mathrm{PBH})$ et de Bonnes Pratiques de Fabrication (BPF). Elles doivent donc s'approprier ces règles afin d'améliorer la qualité du produit. La recherche scientifique doit également travailler la mise au point des séchoirs adaptés au séchage des cossettes de manioc.

\section{Conclusion}

Cette étude a permis de mettre en exergue les conditions de production des cossettes de manioc ainsi que la qualité physico-chimique et microbiologique de cette denrée dans la commune de Bassila. L'enquête effectuée prouve que cette activité est exclusivement réalisée par des femmes, des groupes socioculturels et sociolinguistique Koura et Kotokoli. Ces productrices sont pour la plupart de la trentaine à la quarantaine d'âge en moyenne et ont niveau d'instruction primaire ou non scolarisées. L'évaluation des conditions de production a révélé que les technologie utilisées sont artisanales et ne sont pas de nature à favoriser une bonne qualité du produit fini. Ainsi, certains échantillons présentent une teneur en eau supérieure à $12 \%$, valeur seuil fixée par la Norme Béninoise. D'autres encore présentent des caractéristiques microbiologiques non satisfaisantes et leur consommation peut être à l'origine d'infection, de toxi-infection ou d'intoxination alimentaire.

$\mathrm{Au}$ vue de tout ce qui précède, il urge d'améliorer la technologie de production et de conservation des cossettes, afin de préserver la santé publique. Aussi, les autorités compétentes doivent prendre les mesures idoines pour atteindre cet objectif. Cela passe par la vulgarisation des règles basiques d'hygiène ainsi que la formation des acteurs du secteur. 


\section{CONFLIT D'INTÉRÊTS}

Tous les auteurs déclarent qu'il n'y a aucun conflit d'intérêts pour la publication de cet article.

\section{CONTRIBUTIONS DES AUTEURS}

TRCK a contribué aux différentes collectes des échantillons sur les différents sites d'études, à l'analyse des échantillons et à la rédaction du manuscrit. SEA a apporté sa contribution dans la rédaction scientifique et dans la correction du projet d'article. GRD a Contribué aux différentes collectes des échantillons et a apporté lui aussi des commentaires sur le document. ED-A a apporté sa contribution dans les analyses de laboratoire, dans la rédaction scientifique et dans la correction du projet d'article.

\section{REFERENCES}

Amadi JE, Adebola, MO. 2008. Effect of moisture content and storage conditions on the storability of gari. African Journal of Biotechnology, 7(24). 45914594.

AOAC. 1990. Official methods of analysis (17th edn). AOAC: Arlington, Washington D.C.; 882-883.

AOAC. 1984. Official methods of analysis. AOAC: Arlington, Bénin ;16p.

Biaou G, Monhouanou J, Ahanchede A. 2006. Evaluation interne globale des performances et des résultats du Programme de Développement des Plantes à Racines et Tubercules (PDRT), Volume 1, version provisoire, $128 \mathrm{p}$.

Djoulde, DR, Essia, N G, Etoa, F X. 2007. Nutritive value, toxicological and hygienic quality of some cassava based products consumed in Cameroon. Pakistan Journal of Nutrition 6(4): 404408.

DOI:

10.3923/pjn.2007.404.408

Essono G, Ayodele M, Akoa A, Foko J, Olembo S, Gockowski J. 2007. Aspergillus species on cassava chips in storage in rural areas of southern Cameroon: their relationship with storage duration, moisture content and processing methods. African Journal of Microbiology Research, 1(1): 1-8.

Gnonlonfin GJB, Hell K, Fandohan P, Siame, AB. 2008. Mycoflora and natural occurrence of aflatoxins and fumonisin B 1 in cassava and yam chips from Benin, West Africa. International Journal of Food Microbiology, 122(1) : 140-147.

ISO 6579, Microbiologie des aliments -Méthode horizontale pour la recherche des Salmonella spp, 2002.

Konfo CT, Chabi NW, Agbadjizo J, Dahouenon-Ahoussi E, Soumanou MM, Sohounhloue DC. 2014. Influence de la feuille de Hemizygia bracteosa (Benth) sur la qualité de la bière du sorgho" tchakpalo" produite au Bénin/[Influence of Hemizygia bracteosa (Benth) sheet on the quality of sorghum beer" tchakpalo" produced in Benin]. International Journal of Innovation and Applied Studies, 7(2) : 453-463.

MAEP (2007). Annuaire de la statistique : campagne 2016-2017. Cotonou.

Rasoanantoandro-Gothard MC, Boukar JJL, Floret SC. 2003. L'amélioration des techniques de transformation du manioc en cossettes en RCA : rouissage en vase clos et séchage en encage thermique, Cirad - Prasac, p. 6.

Mukandila KP, Hell K, Hauser S, Lamboni L, Masimango JT. 2012. Qualité des produits dérivés du manioc prélevés au niveau des sites de fabrication et dans les marchés de Kinshasa, RD Congo.

NF EN ISO 6888-1/A1, "Microbiologie des aliments - Méthode horizontale pour le dénombrement des staphylocoques à coagulase positive (Staphylococcus aureus et autres espèces) " - Partie 1 : technique utilisant le milieu gélosé de Baird-Parker-Amendement 1 : inclusion des données de fidélité, 2004.

NF V08-050, "Food microbiology. Coliform counts by counting the colonies obtained at $30^{\circ} \mathrm{C}$ ", Routine method, 1999.

NF V08-051, "Food Microbiology. Enumeration of Microorganisms by Counting the Colonies Obtained at $30^{\circ} \mathrm{C}$ ", Routine method, 1999.

NF V08-059, "Food Microbiology. Enumeration of Yeasts and Molds by Counting Colonies at $25^{\circ} \mathrm{C}$, Routine method, 2002.

NF ISO 7251 (juillet 2005) Microbiologie des aliments - Méthode horizontale pour le 
dénombrement d'Escherichia coli présumés - Technique du nombre le plus probable (Indice de classement : V08020).

NF EN ISO 6579. 2002. Microbiologie des aliments - Méthode horizontale pour la recherche des Salmonella spp. (Indice de classement: V08-013).

NF ISO 15213. 2003. Microbiologie des aliments - Méthode horizontale pour le dénombrement des bactéries sulfitoréductrices se développant en conditions anaérobies (Indice de classement : V08029).

Soulé BG, Aboudou F, Gansari S, Tassou M, Yallou JD. 2013. Analyse de la structure et la dynamique de la chaîne de valeur du manioc au Bénin. Rapport d'étude réalisé par le Laboratoire d'Analyse Régionale et d'Expertise Sociale. LARES, Cotonou, Bénin.

Soule BG, Gansari, Sanni GM. 2008. Bénin : étude sur la commercialisation des produits derives du manioc vers les marches des pays limitrophes (Niger, Nigeria, Togo et Burkina Faso). Initiative regionale pour la transformation et la commercialisation $\mathrm{du}$ manioc (IRTCM). Projet de developpement de racines et tubercules (PDRT). Rapport final. P.78.

Diallo Y, Gueye MT, Sakho M, Darboux PG, Kane A, Barthelemy JP, Lognay G. 2013. Importance nutritionnelle du manioc et perspectives pour l'alimentation de base au Sénégal (synthèse bibliographique)/Nutritional importance of cassava and perspectives as a staple food in Senegal. A review. Biotechnologie, Agronomie, Société et Environnement, 17(4) : 634.

Gmakouba T, Koussao S, Traore E. R, Kpemoua K. E, Zongo J D. 2018. Analyse de la diversité agromorphologique d'une collection de manioc (Manihot esculenta Crantz) du
Burkina Faso. International Journal of Biological and Chemical Sciences, 12(1) : 402-421.

Faiveley M. 2012. L'eau et la conservation des aliments. https://www.techniquesingenieur.fr/base-

documentaire/procedes-chimie-bio-agroth2/biochimie-alimentaire-analyses-etalimentation-humaine-42470210/l-eauet-la-conservation-des-aliments-f1011/

Karidioul D, Akmel DC, Assidjo NE. Trokourey A. 2018. Modélisation du séchage solaire de fèves de cacao par le Réseau de Neurones Artificiel. International Journal of Biological and Chemical Sciences, 12(1): 195-202. DOI: https://dx.doi.org/10.4314/ijbcs.v12i1.15

Gbaguidi-Darboux P, Kouazoundé G, Chabi China LA, Aruna W, Mestres C. 2001. Caractéristiques de la production du lafou au Bénin. In : Technologies postrécolte pour le développement. Actes de l'atelier scientifique, juillet 2001, pp 175-182.

Darman RD. 2004. Mise au point d'un ferment mixte destine à la bioconversion des tubercules de manioc cyanogene (Doctoral dissertation, Université de Ngaoundéré).

Abou M, Yabi I, Yolou I, Ogouwale E. 2018. Caractérisation des systèmes de production sur les sites d'aménagements hydro-agricoles dans le doublet DangboAdjohoun au sud du Bénin. International Journal of Biological and Chemical Sciences, 12(1): 462-478. DOI: https://dx.doi.org/10.4314/ijbcs.v12i1.36

Millogo V, Sissao M, Ouedraogo GA. 2018. Qualité nutritionnelle et bactériologique des échantillons de quelques produits laitiers locaux de la chaîne de production au Burkina Faso. International Journal of Biological and Chemical Sciences, 12(1): 244-252. DOI: https://dx.doi.org/10.4314/ijbcs.v12i1.19 